American Journal of Applied Sciences 8 (12): 1322-1327, 2011

ISSN 1546-9239

(C) 2011 Science Publications

\title{
Hybrid Traffic Management Model for Multi-Protocol Label Switching Network
}

\author{
${ }^{1}$ E.R. Naganathan and ${ }^{2}$ S. Rajagopalan \\ ${ }^{1}$ Department of Computer Application, \\ Velammal Engineering College, Chennai, India \\ ${ }^{2}$ Departement of CSE, Alagappa University, Karaikudi, India
}

\begin{abstract}
Problem statement: MPLS is a highly scalable, protocol agnostic and data-carrying mechanism. Traffic engineering is the major research area in MPLS due to the emerging requirements of MPLS and the internet usage. A major objective of traffic engineering is to minimize or eliminate high-loss situations. Another goal of traffic engineering is to balance the Quality of Service (QoS) against the cost of operating and maintaining the network. Approach: This study proposes a hybrid model for effective traffic management in the MPLS network which contains two models namely traffic flow analysis model and ant colony optimization based routing model. The traffic analysis model will analyses the traffic pattern and identifies the low load path. And the ACO is used for effective transmission in the low load path. Results: The proposed HTM provides lesser packet loss and less response time than existing routing protocol. Conclusion: This hybrid model enables traffic free environment in the congested MPLS network. The routing packet size of the proposed HTM is higher than ACO but comparing existing traditional routing protocol like OSPF and RIP, the packet size is very less.
\end{abstract}

Key words: Quality of Service (QoS), Multi-Protocol Label Switching (MPLS), traffic management, ant colony optimization, hybrid traffic management model, Asynchronous Transfer Mode (ATM)

\section{INTRODUCTION}

Multi-Protocol Label Switching (MPLS) is a mechanism which is used in high-performance telecommunications networks that directs and carries data from one network node to the next with the help of labels. MPLS operates at an OSI Model layer that is generally considered to lie between traditional definitions of Layer 2 and Layer 3 and thus is often referred to as a "Layer 2.5" protocol, which is shown in the Fig. 1. It was designed to provide a unified datacarrying service for both circuit-based clients and packet-switching clients which provide a datagram service model. It can be used to carry many different kinds of traffic, including IP packets, as well as native ATM, SONET and Ethernet frames. MPLS is currently replacing some of these technologies in the marketplace. It is highly possible that MPLS will completely replace these technologies in the future, thus aligning these technologies with current and future technology needs.
MPLS was originally proposed by a group of engineers from IPSILON Networks, but their "IP Switching" technology, which was defined only to work over ATM, did not achieve market dominance. Cisco Systems, Inc., introduced a related proposal, not restricted to ATM transmission, called "Tag Switching". It was a Cisco proprietary proposal and was renamed "Label Switching". It was handed over to the IETF for open standardization. The IETF work involved proposals from other vendors and development of a consensus protocol that combined features from several vendors' work.

This ensures end-to-end circuits over ANY type of transport medium using ANY network layer protocol. In view of the fact that MPLS supports Internet Protocol revised versions (4 and IPv6), IPX, AppleTalk at Layer3; Ethernet, Token Ring, Fiber Distributed Data Interface (FDDI), Asynchronous Transfer Mode (ATM), Frame Relay and Point to Point Protocol (PPP) at Layer 2, it is referred as 'Layer 2.5 protocol'.

Corresponding Author: E.R. Naganathan, Department of Computer Application, Velammal Engineering College, Chennai, India Tel: +9443978509 
Am. J. Applied Sci., 8 (12): 1322-1327, 2011

Traffic, at the packet level for different applications, tends to have different characteristics. This fact has been observed for emerging applications such as video conferencing, peer-topeer and multimedia applications. Moreover, heterogeneous traffic streams are multiplexed together to share the same link. When traffic of different characteristics is multiplexed together, traffic distortion occurs which can be significant depending on the characteristics of the streams MPLS (multi-protocol label switching) network with label-switched paths, potentially for different heterogeneous streams. If multiple paths are being aggregated to share bandwidth, then all of such paths together would be considered as a tunnel. The tunnel is the unit of flow to which a certain bandwidth is allocated at each router that it traverses. In order to minimize distortion, a possible approach would be to classify and multiplex appropriate streams into tunnels so that distortion is minimized within a tunnel. That is, "like-minded" streams can avoid (or minimize) distortion if the network has the capability to do so. For this purpose, one can consider the MPLS network in which multiple Label-Switched Path (LSP) tunnels can be set up between source and destination nodes; such tunnels can be used to ensure logical separation between streams in order to minimize distortions.

Traffic engineering is a method of optimizing the performance of a telecommunications network by dynamically analyzing, predicting and regulating the behavior of data transmitted over that network. The theory of traffic engineering was originally conceived by A.K. Erlang, a Danish mathematician who developed methods of signal traffic measurement in the early 1900s. When message queues become unacceptably long or the frequency of busy signals becomes unacceptably high, the network is said to be in a high-loss condition. A major objective of traffic engineering is to minimize or eliminate high-loss situations. In particular, the number of rejected messages or failed call attempts should be as close to zero as possible. Another goal of traffic engineering is to balance the Quality of Service (QoS) against the cost of operating and maintaining the network.

From the network technology perspective, the integration of the optical layer with electronic layers within a converged data-optical infrastructure, based on classical IP or modern GMPLS (Generalized MPLS) architectures, is a key element in the current trend in broadband network evolution. Two-layer network design problems, where also link and node dimensioning is included in the model, have been considered only quite recently. Some works specifically consider MPLS technology and some of them address the problem of MPLS node location.
Given the complexity of the optimization models, several authors rely on path formulations and column generation coupled with branch and bound, joint column and row generation methods, branch and cut with cut-set inequalities or LP-based decomposition approaches. For mid-to-large networks, the solution of choice remains heuristic algorithms, which provide a feasible solution in limited time. However, to the best of our knowledge, the effect of statistical multiplexing has not been previously considered in such network design and routing models.

Chou (2004) proposed a virtual private network architecture using MPLS, which allows granularity and load balancing. This paper shows feasible result in the view of link utilization, but the multi objective function proposed by the author not optimal in other aspects like response time and packet loss. Radaei and Zukarnain (2009) introduced a distortion factor for heterogeneous streams in traffic engineering of MPLS backbone networks in the presence of tunnelling and capacity constraints by formulating a distortion-aware non-linear discrete optimization problem. The author presented a two-phase heuristic approach to solve this formulation efficiently.

Yasin and Ibrahim (2010) explains the MPLS hierarchical architecture for label-switched networks can be used to address all required functions of converged/unified networks, from initial IP level authentication and configuration, security, session control, resource reservation, admission control, to quality of service and policy management, enhanced only where necessary to address the idiosyncrasies of the mobile wireless environment. This architecture encompassing mostly Internet Engineering Task Force (IETF) standardized protocols, takes advantage of MPLS flexibility to address wireless-specific requirements such as micro mobility as well as nonwireless specific requirements, such as traffic engineering and quality of service and does not involve specific requirements in the mobile terminal for initiating label-switched paths over the wireless interface and allowing end to end interconnection to the backbone network.

Quintero and Frutos (2009) analysed the performance of a Traffic Engineering (TE) strategy for MPLS based network, which is described in Iovanna et al. (2003) is carried out. Specifically the implementation based on a distributed control plane (Internet-like) has been investigated and realized by means of a test bed where real signaling protocol (RSVP-TE) and routing protocols (OSPF-TE) have been implemented. All these previous works are provided feasibility but optimal result based on the current requirement of the internet users such as lower response time and lesser packet loss. 
Am. J. Applied Sci., 8 (12): 1322-1327, 2011

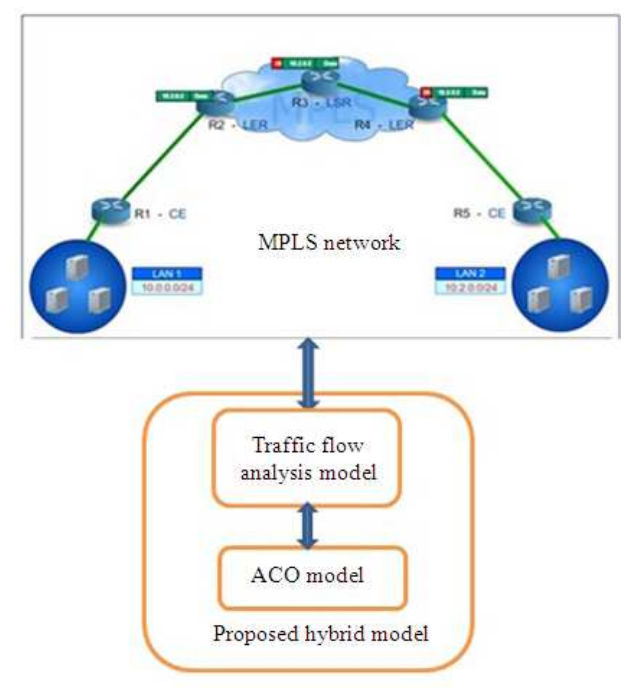

Fig. 1: Proposed hybrid model system design

Mellah and Abbou (2006), the authors propose a mathematical programming model for the design of two-layer networks where MPLS is considered on top of transport networks (SDH or WDM depending on required link speed). This model take into account the tradeoff between the cost of adding MPLS support in the core nodes and the savings in the link bandwidth allocation due to the statistical multiplexing and the traffic grooming effects induced by MPLS nodes. The traffic matrix specifies for each point-to-point request a pair of values: a mean traffic value and an additional one. Using this traffic model, the effect of statistical multiplexing on a link allows allocating a capacity equal to the sum of all the mean values of the traffic demands routed on the link and only the highest additional one. The authors propose a pathbased Mixed Integer Programming (MIP) model for the problem of optimizing the number and location of MPLS nodes in the network and the link capacities. They applied Lagrangian relaxation to this model and use the subgradient method to obtain a lower bound of the network cost. As the number of path variables used to model the routing grows exponentially with the graph size, the authors uses an initially limited number of variables and a column generation approach. Form the results it is observed that the proposed model will suit only for small size and real-world instances.

Mohan and Baskaran (2011a; 2011b), explains a brief survey on ACO in various application of engineering problems (Mohan and Baskaran, 2011a), applied ACO in wired and wireless environment (Mohan and Baskaran, 2011a; 2011b) which reached good result. In our earlier publication (Naganathan et al., 2011), we applied ACO for MPLS network traffic management. The proposed work, applies a traffic flow analysis methodology for identifying current traffic flow and based on the traffic, the proposed model desires the routing. The proposed wok is explained in the following algorithms.

\section{MATERIALS AND METHODS}

Proposed Hybrid Traffic Management (HTM) model: In order to provide highly reliable and congestion free communication in the MPLS network, the traffic management algorithm, to be redefined. In this study, a hybrid model for traffic management is proposed. In the proposed hybrid model contains two models, namely traffic flow analysis model and ant colony based model. The proposed hybrid traffic management model is shown in the Fig. 1. The traffic flow analysis model analyses the traffic pattern of the network traffic in the network and identifies the low load path. This path information is transferred to the Ant Colony Optimization (ACO) based routing model.

Traffic flow analysis model: The proposed traffic flow based routing model is explained in the following algorithm and the ACO based routing model is explained in the algorithm B.

\section{Algorithm for traffic flow analysis model:}

Step 1: For every time interval, $(\Delta t)$, the MPLS periodically propagates hello packets to every other switch connected directly and indirectly in the sub net

Step 2: Each MPLS switch calculates the load factor based on the data flow using the following Eq. 1. Where load factor is the term used to classifies the traffic pattern. In this study, the load factor is classified as three models, which are low traffic, medium traffic and high traffic:

$\mathrm{LFi}=\mathrm{ARi}+(\mathrm{kq} * \mathrm{QLi})) /(\mathrm{LCi} * \Delta \mathrm{ti} * \mathrm{TUi}$

where, LF represents the load factor of MPLS switch ' $i$ ', AR represents the arrival rate of the concern MPLS switch ' $\mathrm{i}$ ', $\mathrm{kq}$ is the constant, QL represents the Queue Length of the concern MPLS switch 'i', LC represents the Link Capacity of the connection established from the concern MPLS switch ' $i$ ' and the TU represents the Target Utility of concern MPLS switch 'i'. 
Step 3: Based on the Load factor, the traffic pattern is classified. If the LF factor of the MPLS switch ' $i$ ' is less than 0.8 then this may be labeled as low traffic. The medium traffic is termed as load factor higher than 0.8 and less than 1 and the high traffic is termed as load factor higher than 1

Step 4: Routing decision is taken based on the load factor of the paths. The path having low traffic is the highly priority route. And the medium traffic path is low priority route. The high traffic path is always avoided in order to avoid traffic

\section{Algorithm for Ant Colony Optimization based routing model:}

\section{//Initialization Phase}

For each pair (r, s), the value of $\tau$

$$
(\mathrm{r}, \mathrm{s}):=\tau
$$

0 End-for

For $\mathrm{k}:=1$ to $\mathrm{m}$ do

Let $(\mathrm{r}, \mathrm{k} 1)$ be the starting city for an ant $\mathrm{k}$

$\operatorname{Jk}(\mathrm{rk} 1):=\{1, \ldots, \mathrm{n}\}-\mathrm{rk} 1$

$/ / \mathrm{Jk}(\mathrm{rk} 1)$ is the set of yet to be

visited cities for //ant $\mathrm{k}$ in city rk1

$\mathrm{rk}:=\mathrm{rk} 1$

$/ / \mathrm{rk}$ is the city where ant $\mathrm{k}$ is located

End-for

//This is the phase in which ants

build their tours. The tour of ant $\mathrm{k}$

is stored in //Tourk

For $\mathrm{i}:=1$ to $\mathrm{n}$ do

$$
\text { If } \mathrm{i}<\mathrm{n}
$$

Then

For $\mathrm{k}:=1$ to $\mathrm{m}$ do

Choose the next city $\mathrm{Sk}$

$\mathrm{Jk}(\mathrm{Sk}):=\mathrm{Jk}(\mathrm{rk})-\mathrm{Sk}$

End-for

$\operatorname{Tourk}(\mathrm{i}):=(\mathrm{rk}, \mathrm{Sk})$

Else

For $\mathrm{k}:=1$ to $\mathrm{m}$ do

//In this cycle all

the ants go back to

the initial city rk1

$\mathrm{Sk}:=\mathrm{rk} 1$ Sk)

Tourk (i) := (rk,

End-for

End-if

//In this phase local updating occurs and pheromone is updated For $\mathrm{k}:=1$ to $\mathrm{m}$ do

$\mathrm{T}(\mathrm{r}, \mathrm{s}) \leftarrow(1-\alpha) \bullet \mathrm{T}(\mathrm{r}, \mathrm{s})+\Sigma(1-\alpha) \bullet \mathrm{T}(\mathrm{r}, \mathrm{s})$ $\mathrm{rk}:=\mathrm{sk} / / \mathrm{New}$ city for ant $\mathrm{k}$
End-for

End-for

//In this phase global updating occurs and pheromone is updated For $\mathrm{k}:=1$ to $\mathrm{m}$ do

Compute Lk // Lk is the length of the tour done by ant $\mathrm{k}$

End-for

Compute Lbest

/*Update edges belonging to Lbest

For each edge $(\mathrm{r}, \mathrm{s})$

$\mathrm{T}(\mathrm{r}, \mathrm{s}) \leftarrow(1-\alpha) \cdot \mathrm{T}(\mathrm{r}, \mathrm{s})+\Sigma(1-\alpha) \bullet \mathrm{T}(\mathrm{r}, \mathrm{s})$

End-for

//In this phase assigns priority

For $\mathrm{k}:=1$ to $\mathrm{m}$ do

Sort the routing table based on pheromone values

\{

Assigns high priority to higher pheromone density path

Choose the best path based on priority in

the wired network and availability and

priority in the case of wireless network

\}

End-for

This proposed hybrid traffic management model is simulated in the NS2. The result and performance analysis is described in the following section.

\section{RESULTS}

The Table 1-6, Fig. 2-4 represents the packet loss, response time in various load condition in User Datagram Protocol (UDP). The Table 7 represents the routing packet size of various routing protocol in bytes.

\begin{tabular}{llcll}
\multicolumn{5}{l}{ Table 1: Packet loss (number of packets) in UDP under normal load } \\
\hline & Normal load & & \\
\cline { 2 - 5 } No of nodes & OSPF & RIP & ACO & HTM \\
\hline 10 & 1 & 1 & 0 & 0 \\
30 & 3 & 1 & 0 & 0 \\
50 & 5 & 2 & 2 & 0 \\
100 & 5 & 19 & 3 & 0 \\
200 & 8 & 22 & 4 & 1 \\
\hline
\end{tabular}

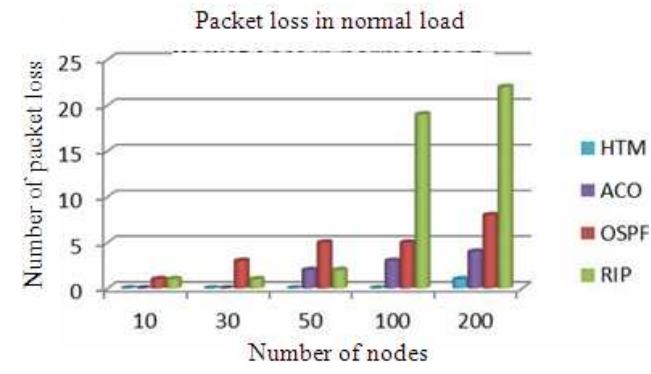

Fig. 2: Packet loss in normal load (number of packets) in UDP 
Am. J. Applied Sci., 8 (12): 1322-1327, 2011

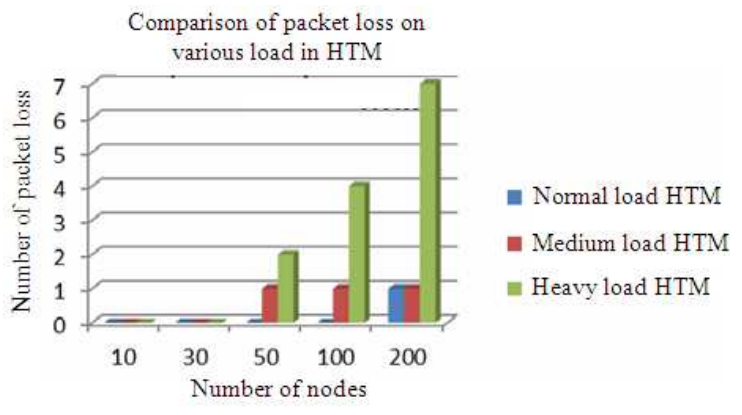

Fig. 3: Comparison of packet loss on various loads in UDP

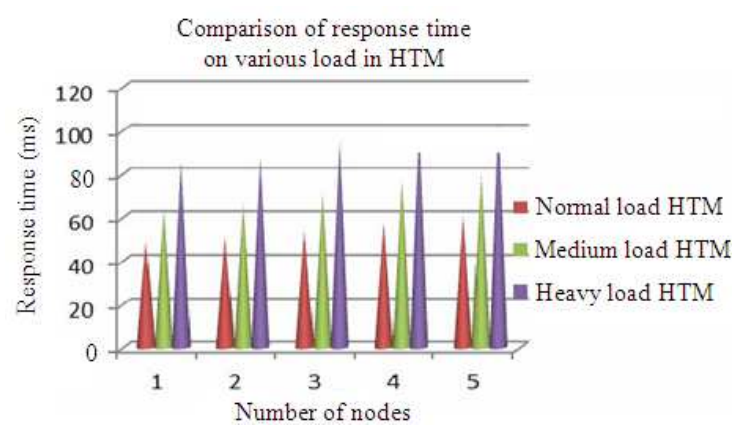

Fig. 4: Comparison of response time on various loads in UDP

Table 2: Packet loss (number of packets) in UDP under medium load Medium load

\begin{tabular}{lcrll} 
& & & & \\
No of nodes & OSPF & RIP & ACO & HTM \\
\hline 10 & 2 & 1 & 1 & 0 \\
30 & 2 & 1 & 1 & 0 \\
50 & 5 & 4 & 3 & 1 \\
100 & 8 & 35 & 5 & 1 \\
200 & 12 & 40 & 8 & 1 \\
\hline
\end{tabular}

Table 3: Packet loss (number of packets) in UDP under heavy load

\begin{tabular}{|c|c|c|c|c|}
\hline \multirow[b]{2}{*}{ No of nodes } & \multicolumn{4}{|c|}{ Heavy load } \\
\hline & OSPF & RIP & $\mathrm{ACO}$ & HTM \\
\hline 10 & 3 & 2 & 1 & 0 \\
\hline 30 & 3 & 2 & 1 & 0 \\
\hline 50 & 4 & 6 & 1 & 2 \\
\hline 100 & 14 & 63 & 12 & 4 \\
\hline 200 & 44 & 72 & 22 & 7 \\
\hline
\end{tabular}

Table 4: Response time (ms) in UDP under normal load

\begin{tabular}{lllll}
\hline & Normal load & & & \\
No of nodes & OSPF & RIP & ACO & HTM \\
\hline 10 & 62 & 58 & 53 & 49 \\
30 & 66 & 63 & 55 & 51 \\
50 & 74 & 71 & 59 & 54 \\
100 & 78 & 79 & 62 & 58 \\
200 & 82 & 90 & 66 & 60 \\
\hline
\end{tabular}

Table 5: Response time (ms) in UDP under medium load

\begin{tabular}{lcrll}
\hline & Medium load & & \\
No of nodes & OSPF & RIP & ACO & HTM \\
\hline 10 & 82 & 77 & 70 & 64 \\
30 & 87 & 84 & 73 & 66 \\
50 & 98 & 94 & 79 & 71 \\
100 & 103 & 105 & 82 & 77 \\
200 & 108 & 119 & 87 & 80 \\
\hline
\end{tabular}

Table 6: Response time (ms) in UDP under heavy load

\begin{tabular}{|c|c|c|c|c|}
\hline \multirow[b]{2}{*}{ No of nodes } & \multicolumn{4}{|c|}{ Heavy load } \\
\hline & OSPF & RIP & $\mathrm{ACO}$ & HTM \\
\hline$\overline{10}$ & 108 & 101 & 92 & 86 \\
\hline 30 & 115 & 111 & 97 & 88 \\
\hline 50 & 129 & 124 & 104 & 95 \\
\hline 100 & 136 & 138 & 108 & 101 \\
\hline 200 & 143 & 157 & 115 & 105 \\
\hline \multicolumn{5}{|c|}{ Table 7: Routing packet size (bytes) } \\
\hline OSPF & \multicolumn{2}{|c|}{ RIP } & $\mathrm{ACO}$ & HTM \\
\hline 44 & \multicolumn{2}{|c|}{44} & 8 & 16 \\
\hline
\end{tabular}

\section{DISCUSSION}

The performance are evaluated using simulation. The proposed work is simulated in NS2. Various Network types with varying number of nodes such as 10 , 30, 50, 100 and 200 number of nodes are designed in the simulator. And various routing protocols such as OSPF, RIP, ACO and proposed HTM is simulated in normal load, medium load and heavy load condition in both TCP and UDP. The simulation is designed for 10sec and the average for $1 \mathrm{sec}$ is recoeded in the given results.

\section{CONCLUSION}

From the result, it is identified that the proposed HTM provides lesser packet loss and less response time than existing routing protocol. The traffic flow analysis is identified the low load path which has lesser traffic flow in their history. The ACO transmit the data using the suggestion given by the traffic flow analysis model. This hybrid model enables traffic free environment in the congested MPLS network. The routing packet size of the proposed HTM is higher than ACO but comparing existing traditional routing protocol like OSPF and RIP, the packet size is very less. Therefore, the proposed HTM is always better than existing routing protocols.

\section{REFERENCES}

Chou, C.T., 2004. Traffic engineering for MPLS-based virtual private networks. Comput. Netw., 44: 319333. DOI: 10.1016/j.comnet.2003.10.001 
Iovanna, P., R. Sabella and M. Settembre, 2003. A traffic engineering system for multilayer networks based on the GMPLS paradigm. IEEE Netw., 17: 28-37. DOI: 10.1109/MNET.2003.1188284

Mellah, H. and F.M. Abbou, 2006. MPLS over segmented WDM optical packet switching networks. J. Comput. Sci., 2: 431-433. DOI: 10.3844/jcssp.2006.431.433

Mohan, B.C. and R. Baskaran, 2011a. Priority and compound rule based routing using ant colony optimization. Int. J. Hybrid Intell. Syst., 8: 93-97.

Mohan, B.C. and R. Baskaran, 2011b. Energy aware and energy efficient routing protocol for adhoc network using restructured artificial bee colony system, High Perform. Archit. Grid Comput., 169: 473-484. DOI: 10.1007/978-3-642-22577-2_65

Naganathan, E.R., S. Rajagopalan and P.H. Raj, 2011. traffic flow analysis model based routing protocol for multi-protocol label switching network. J. Comput. Sci., 7: 1674-1678. DOI: 10.3844/jcssp.2011.1674.1678
Quintero, A. and E.D. Frutos, 2009. MPLS based architecture for mobility and end-to-end QoS support in fourth generation mobile networks. J. Comput. Sci., 5: 255-262. DOI: 10.3844/jcssp.2009.255.262

Radaei, T.A.A. and Z.A. Zukarnain, 2009. Comparison study of transmission control protocol and user datagram protocol behavior over multi-protocol label switching networks in case of failures. J. Comput. Sci., 5: 1042-1047. DOI: 10.3844 jessp.2009.1042.1047

Yasin, W. and H. Ibrahim, 2010. Improving triple play services using multi protocol label switching technology. J. Comput. Sci., 6: 269-278. DOI: 10.3844/jcssp.2010.269.278 